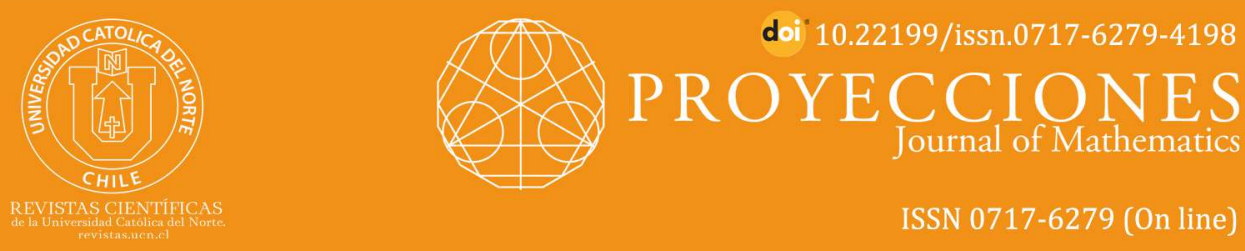

\title{
New types of locally connected spaces via clopen set
}

Ennis Rafael Rosas Rodríguez ${ }^{1}$ () orcid.org/0000-0001-8123-9344 Sarhad F. Namiq ${ }^{2}$ (1) orcid.org/0000-0001-8747-2542

${ }^{1}$ Universidad de la Costa, Dept. de Ciencias Naturales y Exactas, Barranquilla, Colombia. - ennisrafael@gmail.com

${ }^{2}$ University of Garmian, Dept. of Mathematics, Kurdistan, Kalar, Iraq.

⿶sarhad1983@gmail.com

Received: 20 May 2020 | Accepted: 15 January 2021

\section{Abstract:}

In this paper, we define and study a new type of connected spaces called $\lambda_{c o}$ -connected space. It is remarkable that the class of $\lambda$-connected spaces is a subclass of the class of $\lambda_{c o}$-connected spaces. We discuss some characterizations and properties of $\lambda_{c o}$-connected spaces, $\lambda_{c o}$ components and $\lambda_{c o}$-locally connected spaces.

Keywords: $\lambda_{\text {co }}$-connected spaces; $\lambda_{c o}$-components; $\lambda_{\text {co }}$-locally connected spaces.

MSC (2020): 54A05, 54D30.

\section{Cite this article as (IEEE citation style):}

E. R. Rosas Rodríguez and S. F. Namiq, "New types of loca- 


\section{Introduction}

Following [3] N. Levine, 1963, defined semi open sets. Similarly, S. F. Namiq [4], defined an operation $\lambda$ on the family of semi open sets in a topological space called semi operation, denoted by s-operation; via this operation, in his study [7], he defined $\lambda_{s c^{-o p e n}}$ set by using $\lambda$-open and semi closed sets, and also following [5], he defined $\lambda_{c o}$-open set and investigated several properties of $\lambda_{c o}$-derived, $\lambda_{c o}$-interior and $\lambda_{c o}$-closure points in topological spaces.

In the present article, we define the $\lambda_{c o}$-connected space, discuss some characterizations and properties of $\lambda_{c o}$-connected spaces, $\lambda_{c o}$-components and $\lambda_{c o}$-locally connected spaces and finally its relations with others connected spaces.

\section{Preliminaries}

In the entire parts of the present paper, a topological space is referred to by $(X, \tau)$ or simply by $X$. First, some definitions are recalled and results are used in this paper. For any subset $A$ of $X$, the closure and the interior of $A$ are denoted by $\mathrm{Cl}(A)$ and $\operatorname{Int}(A)$, respectively. Following [8], the researchers state that a subset $A$ of $X$ is regular closed if $A=\mathrm{Cl}(\operatorname{Int}(A))$. Similarly, following [3], a subset $A$ of a space $X$ is semi open if $A \subseteq \mathrm{Cl}(\operatorname{Int}(A))$. The complement of a semi open set is called semi closed. The family of all semi open (resp. semi closed) sets in a space $X$ is denoted by $\mathrm{SO}(X, \tau)$ or $\mathrm{SO}(X)$ (resp. $\operatorname{SC}(X, \tau)$ or $\operatorname{SC}(X)$. According to [1], a space $X$ is stated to be sconnected, if it is not the union of two nonempty disjoint semi open subsets of $X$. We consider $\lambda: \mathrm{SO}(X) \rightarrow P(X)$ as a function defined on $\mathrm{SO}(X)$ into the power set of $X, P(X)$ and $\lambda$ is called a semi-operation denoted by s-operation, if $V \subseteq \lambda(V)$, for each semi open set $V$. It is assumed that $\lambda(\emptyset)=\emptyset$ and $\lambda(X)=X$, for any s-operation. Let $X$ be a space and $\lambda$ $: \mathrm{SO}(X) \rightarrow P(X)$ be an s-operation, following [4], a subset $A$ of $X$ is called a $\lambda$-open set, which is equivalent to $\lambda_{s}$-open set [2], if for each $x \in A$, there exists a semi open set $U$ such that $x \in U$ and $\lambda(U) \subseteq A$. The complement of a $\lambda$-open set is called a $\lambda$-closed. The family of all $\lambda$-open (resp., $\lambda$-closed) subsets of a space $X$ is denoted by $\mathrm{SO}_{\lambda}(X, \tau)$ or $\mathrm{SO}_{\lambda}(X)\left(\operatorname{resp}, \mathrm{SC}_{\lambda}(X, \tau)\right.$ or $\left.\mathrm{SC}_{\lambda}(X)\right)$. Following [4], a $\lambda$-open subset $A$ of $X$ is named a $\lambda_{c^{-}}$-open set, if for each $x \in A$, there exists a closed set $F$ such that $x \in F \subseteq A$. The family of all $\lambda_{c}$-open (resp., $\lambda_{c}$-closed ) subsets of a space $X$ is denoted by $\mathrm{SO}_{\lambda_{c}}(X, \tau)$ or $\mathrm{SO}_{\lambda_{c}}(X)$ (resp, $\mathrm{SC}_{\lambda_{c}}(X, \tau)$ or $\mathrm{SC}_{\lambda_{c}}(X)$ ). Thus, a number of 
definitions are presented and some known results are reiterated which will be used in the sequel.

Definition 2.1. [4] Let $X$ be a space and $\lambda: S O(X) \rightarrow P(X)$ be an $s^{-}$ operation, then a subset $A$ of $X$ is called a $\lambda$-open set if for each $x \in A$ there exists a semi open set $U$ such that $x \in U$ and $\lambda(U) \subseteq A$. The complement of a $\lambda$-open set is called $\lambda$-closed. The family of all $\lambda$-open (resp., $\lambda$-closed) subsets of a topological space $(X, \tau)$ is denoted by $S_{\lambda}(X, \tau)$ or $S_{\lambda}(X)$ ( resp., $S C_{\lambda}(X, \tau)$ or $\left.S C_{\lambda}(X)\right)$.

Definition 2.2. [5] $A \lambda$-open subset $A$ of $X$ is called a $\lambda_{c o}$-open (resp., $\lambda_{c}$-open [4]) set if for each $x \in A$, there exists a clopen (resp., closed) set $F$ such that $x \in F \subseteq A$. The family of all $\lambda_{c}$-open (resp., $\lambda_{c}$-closed) subsets of a space $X$ is denoted by $S O_{\lambda_{c}}(X, \tau)$ or $S O_{\lambda_{c}}(X)$ (resp $S C_{\lambda_{c}}(X, \tau)$ or $\left.S C_{\lambda_{c}}(X)\right)$. The family of all $\lambda_{c o}$-open (resp., $\lambda_{c o}$-closed) subsets of a space $X$ is denoted by $S O_{\lambda_{c o}}(X, \tau)$ or $S O_{\lambda_{c o}}(X)\left(\operatorname{resp} S C_{\lambda_{c o}}(X, \tau)\right.$ or $\left.S C_{\lambda_{c o}}(X)\right)$.

Proposition 2.3. [4],[5] For a space $X, S O_{\lambda_{c o}}(X) \subseteq S O_{\lambda_{c}}(X) \subseteq S O_{\lambda}(X) \subseteq S O(X)$.

Definition 2.4. [2] Let $X$ be a space, an s-operation $\lambda$ is said to be sregular if for every semi open sets $U$ and $V$ containing $x \in X$, there exists a semi open set $W$ containing $x$ such that $\lambda(W) \subseteq \lambda(U) \cap \lambda(V)$.

Definition 2.5. [6] $A$ space $X$ is said to be $\lambda$-connected if there does not exist a pair $A, B$ of nonempty disjoint $\lambda$-open subset of $X$ such that $X=A \cup B$, otherwise $X$ is called $\lambda$-disconnected. In this case, the pair $(A, B)$ is called a $\lambda$-disconnection of $X$.

Following [5], we used some results:

Definition 2.6. Let $X$ be a space and $A$ a subset of $X$. Then:

1. The $\lambda_{c o}$-closure of $A$, denoted by $\lambda_{c o} C l(A)$ is the intersection of all $\lambda_{c o}$-closed sets containing $A$.

2. The $\lambda_{c o}$-interior of $A$, denoted by $\lambda_{c o} \operatorname{Int}(A)$ is the union of all $\lambda_{c o^{-}}$ open sets of $X$ contained in $A$.

3. A point $x \in X$ is said to be a $\lambda_{c o}$-limit point of $A$ if every $\lambda_{c o}$-open set containing $x$ contains a point of $A$ different from $x$, and the set of all $\lambda_{c o}$-limit points of $A$ is called the $\lambda_{c o}$-derived set of $A$, denoted by $\lambda_{c o} D(A)$. 
Proposition 2.7. For each point $x \in X, x \in \lambda_{c o} C l(A)$ if and only $V \cap A \neq$ $\emptyset$, for every $V \in S O_{\lambda_{c o}}(X)$ such that $x \in V$.

Proposition 2.8. Let $\left\{A_{\alpha}\right\}_{\alpha \in I}$ be any collection of $\lambda_{c o}$-open sets in a topological space $(X, \tau)$, then $\cup_{\alpha \in I} A_{\alpha}$ is a $\lambda_{c o}$-open set.

Example 2.9. Let $X=\{a, b, c\}$ and $\tau=P(X)$. We define an s-operation $\lambda: S O(X) \rightarrow P(X)$ as:

$$
\lambda(A)=\left\{\begin{array}{l}
A \text { if } A \neq\{a\},\{b\} \\
X \quad \text { otherwise }
\end{array}\right.
$$

Now, we have $\{a, b\}$ and $\{b, c\}$ are $\lambda_{c o}$-open sets, but $\{a, b\} \cap\{b, c\}=\{b\}$ is not $\lambda_{c o}$-open.

Proposition 2.10. Let $\lambda$ be an s-operation and s-regular. If $A$ and $B$ are $\lambda_{c o}$-open sets in $X$, then $A \cap B$ is also a $\lambda_{c o}$-open set.

Proposition 2.11. Let $X$ be a space and $A \subseteq X$. Then $A$ is a $\lambda_{c o}$-closed subset of $X$ if and only if $\lambda_{c o} D(A) \subseteq A$.

Proposition 2.12. For subsets $A, B$ of a space $X$, the following statements are true.

1. $A \subseteq \lambda_{c o} C l(A)$.

2. $\lambda_{c o} C l(A)$ is a $\lambda_{c o}$-closed set in $X$.

3. $\lambda_{c o} C l(A)$ is a smallest $\lambda_{c o}$-closed set, containing $A$.

4. $A$ is a $\lambda_{c o}$-closed set if and only if $A=\lambda_{c o} C l(A)$.

5. $\lambda_{c o} C l(\emptyset)=\emptyset$ and $\lambda_{c o} C l(X)=X$.

6. If $A$ and $B$ are subsets of the space $X$ with $A \subseteq B$. Then $\lambda_{c o} C l(A) \subseteq$ $\lambda_{c o} C l(B)$.

7. For any subsets $A, B$ of a space $X$. $\lambda_{c o} C l(A) \cup \lambda_{c o} C l(B) \subseteq \lambda_{c o} C l(A \cup$ $B)$.

8. For any subsets $A, B$ of a space $X . \quad \lambda_{c o} C l(A \cap B) \subseteq \lambda_{c o} C l(A) \cap$ $\lambda_{c o} C l(B)$.

Proposition 2.13. Let $X$ be a space and $A \subseteq X$. Then $\lambda_{c o} C l(A)=$ $A \cup \lambda_{c o} D(A)$. 


\section{3. $\lambda_{c o}$-Connected Spaces}

In this section, we define, study and characterize the $\lambda_{c o}$-connected space, finally some of its properties are established.

We start this section with the following definitions.

Definition 3.1. Let $X$ be a space and $Y \subseteq X$. Then the class of $\lambda_{c o}$-open sets in $Y$ denoted by $S O_{\lambda_{c o}}(Y)$, is defined in a natural way as: $S O_{\lambda_{c o}}(Y)=$ $\left\{Y \cap V: V \in S O_{\lambda_{c o}}(X)\right\}$. That is, $W$ is $\lambda_{c o}$-open in $Y$ if and only if $W=Y \cap V$, where $V$ is a $\lambda_{c o}$-open set in $X$. Thus, $Y$ is a subspace of $X$ with respect to $\lambda_{c o}$-open set.

Definition 3.2. A space $X$ is said to be $\lambda_{c o}$-connected if there does not exist a pair $A, B$ of nonempty disjoint $\lambda_{\text {co-open subset of } X \text { such that }}$ $X=A \cup B$, otherwise $X$ is called $\lambda_{c o}$-disconnected. In this case, the pair $(A, B)$ is called a $\lambda_{c o}$-disconnection of $X$.

Definition 3.3. Let $X$ be a space and $\lambda: S O(X) \rightarrow P(X)$ an s-operation, then the family $S O_{\lambda_{c o}}(X)$ is called $\lambda_{c o}$-indiscrete space if $S O_{\lambda_{c o}}(X)=$ $\{\emptyset, X\}$.

Definition 3.4. Let $X$ be a space and $\lambda: S O(X) \rightarrow P(X)$ an s-operation then the family $S O_{\lambda_{c o}}(X)$ is called a $\lambda_{c o}$-discrete space if $S O_{\lambda_{c o}}(X)=P(X)$.

Example 3.5. Every $\lambda_{c o}$-indiscrete space is $\lambda_{c o}$-connected.

We give in below a characterization of $\lambda_{c o}$-connected spaces, the proof of which is straight forward.

Theorem 3.6. A space $X$ is $\lambda_{c o}$-disconnected (resp. $\lambda_{c o}$-connected) if and only if there exists (resp., does not exist) a nonempty proper subset $A$ of $X$, which is both $\lambda_{c o}$-open and $\lambda_{c o}$-closed in $X$.

Theorem 3.7. Every $\lambda$-connected space is $\lambda_{c o}$-connected.

Let $X$ be $\lambda$-connected, then there does not exist a pair $A, B$ of nonempty disjoint $\lambda$-open subset of $X$ such that $X=A \cup B$, but every $\lambda_{c o}$-open set is a $\lambda$-open set by Proposition 2.3, so there does not exist a pair $A, B$ of nonempty disjoint $\lambda_{c o}$-open subset of $X$ such that $X=A \cup B$. Thus $X$ is $\lambda_{c o}$-connected.

The converse of Theorem 3.7, is not true in general as it is shown by the following example. 
Example 3.8. Let $X=\{a, b, c\}$, and $\tau=\{\emptyset,\{a\},\{b\},\{a, b\},\{a, c\}, X\}$. We define an s-operation $\lambda: S O(X) \rightarrow P(X)$ as follows:

$$
\lambda(A)=\left\{\begin{array}{c}
A \quad \text { if } A=\{a\}, \\
X \quad \text { otherwise }
\end{array}\right.
$$

$S O(X)=\{\emptyset,\{a\},\{b\},\{a, b\},\{a, c\}, X\}$.

$S O_{\lambda}(X)=\{\emptyset,\{a\}, X\}$.

$S O_{\lambda_{c o}}(X)=\{\emptyset, X\}$.

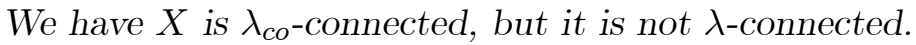

Definition 3.9. Let $X$ be a space and $A \subseteq X$. The $\lambda_{c o}$-boundary of $A$, denoted by $\lambda_{c o} B d(A)$, is defined as the set $\lambda_{c o} B d(A)=\lambda_{c o} C l(A) \cap \lambda_{c o} C l(X \backslash$ A).

Theorem 3.10. A space $X$ is $\lambda_{c o}$-connected if and only if every nonempty proper subspace has a nonempty $\lambda_{c o}$-boundary.

Suppose that a nonempty proper subspace $A$ of a $\lambda_{c o}$-connected space $X$ has empty $\lambda_{c o}$-boundary. Then $A$ is $\lambda_{c o}$-open and $\lambda_{c o} \operatorname{Cl}(A) \cap \lambda_{c o} \operatorname{Cl}(X \backslash A)=$ $\emptyset$. Let $p$ be a $\lambda_{c o}$-limit point of $A$. Then $p \in \lambda_{c o} \mathrm{Cl}(A)$, but $p \notin \lambda_{c o} \mathrm{Cl}(X \backslash A)$. In particular $p \notin(X \backslash A)$ and so $p \in A$. Thus $A$ is $\lambda_{c o}$-closed and $\lambda_{c o}$-open. By Theorem 3.6, $X$ is $\lambda_{c o}$-disconnected. This contradiction gives that $A$ has a nonempty $\lambda_{c o}$-boundary.

Conversely, suppose $X$ is $\lambda_{c o}$-disconnected. Then by Theorem 3.6, $X$ has a proper subspace $A$ which is both $\lambda_{c o}$-closed and $\lambda_{c o}$-open. Then $\lambda_{c o} \mathrm{Cl}(A)=$ $A, \lambda_{c o} \mathrm{Cl}(X \backslash A)=(X \backslash A)$ and $\lambda_{c o} \mathrm{Cl}(A) \cap \lambda_{c o} \mathrm{Cl}(X \backslash A)=\emptyset$. So $A$ has empty $\lambda_{c o}$-boundary, a contradiction. Hence $X$ is $\lambda_{c o}$-connected. This completes the proof.

Theorem 3.11. Let $(A, B)$ be a $\lambda_{c o}$-disconnection of a space $X$ and $C$ be a $\lambda_{c o}$-connected subspace of $X$. Then $C$ is contained in $A$ or in $B$.

Suppose that $C$ is neither contained in $A$ nor in $B$. Then $C \cap A, C \cap B$ are both nonempty $\lambda_{c o}$-open subsets of $C$ such that $(C \cap A) \cap(C \cap B)=\emptyset$ and $(C \cap A) \cup(C \cap B)=C$. This gives that $(C \cap A, C \cap B)$ is a $\lambda_{c o}$-disconnection of $C$. This contradiction proves the theorem.

Theorem 3.12. Let $X=\cup_{\alpha \in I} X_{\alpha}$, where each $X_{\alpha}$ is $\lambda_{c o}$-connected and $\bigcap_{\alpha \in I} X_{\alpha} \neq \emptyset$. Then $X$ is $\lambda_{c o}$-connected. 
Suppose on the contrary that $(A, B)$ is a $\lambda_{c o}$-disconnection of $X$. Since each $X_{\alpha}$ is $\lambda_{c o}$-connected, therefore by Theorem 3.11, $X_{\alpha} \subseteq A$ or $X_{\alpha} \subseteq A$. Since $\bigcap_{\alpha \in I} X_{\alpha} \neq \emptyset$, therefore all $X_{\alpha}$ are contained in $A$ or in $B$. This gives that, if $X \subseteq A$, then $B=\emptyset$ or if $X \subseteq B$, then $A=\emptyset$. This contradiction proves that $X$ is $\lambda_{c o}$-connected. Which completes the proof.

Using Theorem 3.12, we give a characterization of $\lambda_{c o}$-connectedness as follows:

Theorem 3.13. A space $X$ is $\lambda_{c o}$-connected if and only if for every pair of points $x, y$ in $X$, there is a $\lambda_{c o}$-connected subset of $X$, which contains both $x$ and $y$.

The necessity is immediate since the $\lambda_{c o}$-connected space itself contains these two points. For the sufficiency, suppose that for any two points $x, y$; there is a $\lambda_{c o}$-connected subspace $C_{(x, y)}$ of $X$ such that $x, y \in C_{(x, y)}$. Let $a \in X$ be a fixed point and $\left\{C_{(a, x)}: x \in X\right\}$ a class of all $\lambda_{c o}$-connected subsets of $X$, which contain the points $a, x$. Then $X=\cup_{x \in X} C_{(a, x)}$ and $\cap_{x \in X} C_{(a, x)} \neq \emptyset$. Therefore, by Theorem 3.12, $X$ is $\lambda_{c o}$-connected. This completes the proof.

Theorem 3.14. Let $C$ be a $\lambda_{c o}$-connected subset of a space $X$ and $A \subseteq X$ such that $C \subseteq A \subseteq \lambda_{c o} C l(C)$. Then $A$ is $\lambda_{c o}$-connected.

It is sufficient to show that $\lambda_{c o} \mathrm{Cl}(C)$ is $\lambda_{c o}$-connected. On the contrary, suppose that $\lambda_{c o} \mathrm{Cl}(C)$ is $\lambda_{c o}$-disconnected. Then there exists a $\lambda_{c o^{-}}$-disconnection $(H, K)$ of $\lambda_{c o} \mathrm{Cl}(C)$. That is, $H \cap C, K \cap C$ are $\lambda_{c 0^{-}}$ open sets in $C$ such that $(H \cap C) \cap(K \cap C)=(H \cap K) \cap C=\emptyset$ and $(H \cap C) \cup(K \cap C)=(H \cup K) \cap C=C$. This gives that $(H \cap C, K \cap C)$ is a $\lambda_{c o}$-disconnection of $C$, a contradiction. This proves that $\lambda_{c o} \operatorname{Cl}(C)$ is $\lambda_{c o}$-connected.

\section{4. $\lambda_{c o}$-components and $\lambda_{c o}$-locally connected spaces}

In this section a new types of $\lambda_{c o}$-component of a space $X$ and $\lambda_{c o}$-locally connected space are defined, studied and characterized and finally some of its properties are established.

Definition 4.1. A maximal $\lambda_{c o}$-connected subset of a space $X$ is called a $\lambda_{c o}$-component of $X$. If $X$ itself is $\lambda_{c o}$-connected, then $X$ is the only $\lambda_{c o}$-component of $X$. 
Next we study the properties of $\lambda_{c o}$-components of a space $X$.

Theorem 4.2. Let $(X, \tau)$ be a topological space. Then:

1. For each $x \in X$, there is exactly one $\lambda_{c o}$-component of $X$ containing $x$.

2. Each $\lambda_{c o}$-connected subset of $X$ is contained in exactly one $\lambda_{c o^{-}}$ component of $X$.

3. A $\lambda_{c o}$-connected subset of $X$, which is both $\lambda_{c o}$-open and $\lambda_{c o}$-closed is a $\lambda_{c o}$-component, if $\lambda$ is s-regular.

4. Every $\lambda_{c o}$-component of $X$ is $\lambda_{c o}$-closed in $X$.

(1)-Let $x \in X$ and $\left\{C_{\alpha}: \alpha \in I\right\}$ be a class of all $\lambda_{c o}$-connected subsets of $X$ containing $x$. Put $C=\bigcup_{\alpha \in I} C_{\alpha}$, then by Theorem 3.12, $C$ is $\lambda_{c o^{-}}$ connected and $x \in X$. Suppose $C \subseteq C^{1}$, for some $\lambda_{c o}$-connected subset $C^{1}$ of $X$. Then $x \in C^{1}$ and hence $C^{1}$ is one of the $C_{\alpha}$ 's and hence $C^{1} \subseteq C$. Consequently $C=C^{1}$. This proves that $C$ is a $\lambda_{c o}$-component of $X$, which contains $x$.

(2)-Let $A$ be a $\lambda_{c o}$-connected subset of $X$, which is not a $\lambda_{c o}$-component of $X$. Suppose that $C_{1}, C_{2}$ are $\lambda_{c o}$-components of $X$ such that $A \subseteq C_{1}$, $A \subseteq C_{2}$. Since $C_{1} \cap C_{2} \neq \emptyset, C_{1} \cup C_{2}$ is another $\lambda_{c o}$-connected set which contains $C_{1}$ as well as $C_{2}$, this contradicts the fact that $C_{1}$ and $C_{2}$ are $\lambda_{c 0^{-}}$ components. This proves that $A$ is contained in exactly one $\lambda_{c o}$-component of $X$.

(3)-Suppose that $A$ is a $\lambda_{c o}$-connected subset of $X$ which is both $\lambda_{c o}$-open and $\lambda_{c o}$-closed. By (2), $A$ is contained in exactly one $\lambda_{c o}$-component $C$ of $X$. If $A$ is a proper subset of $C$, and since $\lambda$ is s-regular, therefore $C=(C \cap A) \cup(C \cap(X \backslash A))$ is a $\lambda_{c o}$-disconnection of $C$, a contradiction. Thus, $A=C$.

(4)-Suppose a $\lambda_{c o}$-component $C$ of $X$ is not $\lambda_{c o}$-closed. Then, by Theorem $3.14, \lambda_{c o} \mathrm{Cl}(A)$ is $\lambda_{c o}$-connected containing a $\lambda_{c o}$-component $C$ of $X$. This implies $C=\lambda_{c o} \mathrm{Cl}(A)$ and hence $C$ is $\lambda_{c o}$-closed. This completes the proof.

We introduce the following definition

Definition 4.3. A space $X$ is said to be locally $\lambda_{c o}$-connected if for any point $x \in X$ and any $\lambda_{c o}$-open set $U$ containing $x$, there is a $\lambda_{c o}$-connected and $\lambda_{c o}$-open set $V$ such that $x \in V \subseteq U$. 
Theorem 4.4. A $\lambda_{c o}$-open subset of $\lambda_{c o}$-locally connected space is $\lambda_{c o^{-}}$ locally connected.

Let $U$ be a $\lambda_{c o}$-open subset of a $\lambda_{c o}$-locally connected space $X$. Let $x \in U$ and $V$ a $\lambda_{c o}$-open nbd of $x$ in $U$. Then $V$ is a $\lambda_{c o}$-open neighborhood of $x$ in $X$. Since $X$ is $\lambda_{c o^{-}}$-locally connected, therefore there exists a $\lambda_{c o^{-}}$ connected, $\lambda_{c o}$-open neighborhood $W$ of $x$ such that $x \in W \subseteq V$. So that $W$ is also a $\lambda_{c o}$-connected and $\lambda_{c o}$-open neighborhood $x$ in $U$ such that $x \in W \subseteq U \subseteq V$ or $x \in W \subseteq V$. This proves that $U$ is $\lambda_{c o}$-locally connected.

\section{References}

[1] C. Dorsett, "Semi-connectedness", Indian journal of mechanic mathematics, vol. 17, no. 1, pp. 57-63, 1979.

[2] A. B. Khalaf and S. F. Namiq, " $\lambda$-open sets and $\lambda$-separation axioms in topological spaces", Journal of advanced studies in topology, vol. 4, no. 1, pp. 150-158, 2013.

[3] N. Levine, "Semi-open sets and semi-continuity in topological spaces", The american mathematical monthly, vol. 70, no. 1, pp. 36-41, 1963, doi: 10.1080/ 00029890.1963.11990039

[4] S. F. Namiq, "New types of continuity and separation axiom based operation in topological spaces", MSc Thesis, University of Sulaimani, 2011.

[5] S. F. Namiq, "入co-open sets and topological properties", Submit.

[6] S. F. Namiq, " $\lambda$-connected spaces via $\lambda$-open sets", Journal of Garmian University, vol. 7, pp. 165-178, 2015.

[7] S. F. Namiq, "גsc-open sets and topological properties", Journal of Garmian University, 2014. [On line]. Available: https:/ / bit.ly/ 3tucwua

[8] M. H. Stone, "Applications of the theory of boolean rings to general topology", Transactions of the American Mathematical Society, vol. 41, no. 3, pp. 375-375, Mar. 1937, doi: 10.1090/S0002-9947-19371501905-7 